\title{
sciendo
}

\section{Differences in Mechanical Midsole Characteristics of Running Shoes Do Not Influence Physiological Variables in Aerobic and Anaerobic Running}

\author{
by \\ Christian Mitschke ${ }^{1}$, Katrin Karger ${ }^{1}$, Thomas L. Milani ${ }^{1}$
}

\begin{abstract}
The purpose of this study was to determine the influence of footwear stiffness and energy loss on oxygen uptake and heart rate in athletes running under aerobic and anaerobic conditions. Four footwear conditions with identical outsoles, insoles, upper materials, but different mechanical properties regarding polyurethane midsole materials were investigated. Respective midsole material characteristics were selected to represent a wide range of running shoes. The test procedure for eighteen well-trained male runners was divided into three treadmill testing sessions: an incremental ramp protocol to estimate the individual ventilatory threshold (day 1), a test with 6-minute stages in each shoe at $70 \%$ (aerobic, day 2) and 102\% (anaerobic, day 3) of the participant's ventilatory threshold. For oxygen uptake and the heart rate, no significant differences between footwear conditions were found for either running condition. Furthermore, no significant relationships between physiological variables and mechanical midsole characteristics were found. The wide range of significant stiffness differences in the rearfoot $(52.7 \mathrm{~N} / \mathrm{mm})$ and forefoot areas $(50.7 \mathrm{~N} / \mathrm{mm})$, as well as significant differences of the shoe midsole material energy loss in the rearfoot $(18.8 \%)$ and forefoot areas $(10.7 \%)$ were too low to influence physiological variables significantly when running below and slightly above the ventilatory threshold. It seems that shoe mass and shoe comfort can influence physiological variables more than the mechanical midsole characteristics of stiffness and energy loss. These results may have practical implications for shoe manufacturers, coaches, and athletes, alike.
\end{abstract}

Key words: footwear, midsole stiffness, energy loss, running, oxygen uptake, heart rate.

\section{Introduction}

Endurance performance in distance running can be influenced by a variety of factors, such as footwear comfort and running mechanics, e.g. stride length and a foot strike pattern (Cavanagh and Williams, 1982; Luo et al., 2009; Perl et al., 2012). Furthermore, varying theories exist on the influence of the shoe type, mass, and cushioning systems on physiological variables of runners (Burkett et al., 1985; Catlin and Dressendorfer, 1979; Frederick et al., 1980; Nigg et al., 2003; Worobets et al., 2013). Most studies analyze two different shoes under aerobic conditions or at predetermined speeds, and neglect subjects' aerobic thresholds and fitness levels. There are only a few studies investigating the effects of running shoes under anaerobic conditions slightly above the aerobic threshold (Luo et al., 2009; Nigg et al., 2003). A systematic review showed that the effects of mechanical midsole properties on running performance under aerobic and anaerobic conditions have not yet been investigated (Fuller et al., 2015). However, both energy metabolisms are required for middle and long-distance running. The change from steady state oxygen consumption to the anaerobic

\footnotetext{
1 - Department of Human Locomotion, Institute of Human Movement Science E Health, Chemnitz University of Technology, Chemnitz, Saxony, Germany.
} 
metabolism occurs when transitioning from moderate running to high intensity running. This is a typical scenario during interval training sessions and sprint finishes in middle and longdistance races. Therefore, midsole properties should support performance of athletes under aerobic as well as anaerobic conditions by reducing energy expenditure.

Furthermore, studies investigating the influence of mechanical midsole properties on physiological variables measured material characteristics only for the heel region or for general midsole hardness (Frederick et al., 1980; Hardin et al., 2004; Nigg et al., 2003; Worobets et al., 2013). However, high forces act on the forefoot region during the takeoff, which can have an effect on athletes' physiological variables (Nigg and Segesser, 1992; Stefanyshyn and Nigg, 2000). Due to differences in midsole construction, such as height differences between the heel and the forefoot, it cannot be assumed that mechanical properties are constant throughout the midsole. Therefore, the material characteristics of the whole midsole should be considered to correctly interpret the results of the physiological variables.

The purpose of the current study was to analyze the influence of midsole material characteristics of four running shoes on chosen physiological variables. To avoid effects due to differences in shoe mass, fit, cushioning systems, or shoe types (cushioned shoe or competition shoe), the four running shoes differed only in midsole properties. We hypothesized that running in shoes with: a) higher stiffness and b) less energy loss would lead to lower oxygen uptake and a lower heart rate during aerobic and anaerobic running.

\section{Methods}

\section{Participants}

Eighteen well-trained male runners (more than six hours of running training per week) without any injuries in the last six months participated in this study. Yet, only fifteen participants (age: 24.4 \pm 3.5 years, body height: $176.5 \pm 4.4 \mathrm{~cm}$, body mass: $69.5 \pm 3.5 \mathrm{~kg}$, running distance per week: $37.5 \pm 23.1 \mathrm{~km}$, shoe size UK 8) completed all tests successfully. To avoid effects of fatigue in the aerobic and anaerobic test sessions (of approximately 45-minute duration), only welltrained runners were recruited. The participants were given information about the purpose and design of the study. They signed an informed consent document and completed a form with their personalized data. All procedures were performed in accordance with the recommendations of the Declaration of Helsinki. This study was approved by the Ethics Committee of the Faculty of Behavioural and Social Sciences of the Chemnitz University of Technology

(IfS_Mil_Karg_EnergyReturnEffect_290312).

Participants were instructed to avoid strenuous training and physical work one day before and directly before testing, and to eat and drink adequately on testing days.

\section{Measures}

\section{Footwear conditions}

To investigate the influence of sole materials on physiological variables, four different footwear conditions (men's UK size 8) were analyzed in this study. The shoes (PM1 to PM4) were prototypes with identical outsoles, insoles, and upper materials (PUMA Complete Concinnity), but differed only in mechanical properties with regard to their polyurethane midsole materials (Figure 1). Respective midsole material characteristics were selected to represent a wide range of running shoes (hard competition shoe to soft cushioned shoe).

To quantify the differences of the sole materials in the rearfoot and forefoot areas, the shoes were tested in a servo-hydraulic testing device (HC10; Zwick GmbH \& Co. KG; Ulm, Germany) as described in Schwanitz and Odenwald (2008) and in Mitschke et al. (2018). The load-time profile was derived from biomechanical measurements of ground reaction force while running at a velocity of $3.5 \pm 0.1 \mathrm{~m} / \mathrm{s}$ (Brückner et al., 2010). We analyzed the following mechanical variables: midsole stiffness (stiffness) and relative energy loss (ELoss_rel) for the rearfoot and forefoot areas. Stiffness $(\mathrm{N} / \mathrm{mm})$ represents shoe midsole deformation at the maximum load of $1550 \mathrm{~N}$ (rearfoot test) as well as $1750 \mathrm{~N}$, (forefoot test) in relation to shoe midsole deformation at the $1000 \mathrm{~N}$ load (Schwanitz and Odenwald, 2008). Shoes with high stiffness were described as hard, and shoes with lower stiffness were described as compliant. ELoss_rel (\%) represents the percentage ratio of energy loss over energy input. Force and deformation data were 
captured using integrated sensors with a sampling frequency of 1000 frames per second, and data were analyzed using MATLAB R2016a (MathWorksTM, Massachusetts, USA) in post processing.

\section{Physiological variables}

Running sessions were performed on a treadmill $\left(\mathrm{h} / \mathrm{p} / \operatorname{cosmos}{ }^{\circledR}\right.$ quasar $5.0, \mathrm{~h} / \mathrm{p} / \operatorname{cosmos}$ sports \& medical $\mathrm{GmbH}$, Nussdorf-Traunstein, Germany). Oxygen uptake was quantified during running using a MetaLyzer 3B system (CORTEX Biophysik GmbH, Leipzig, Germany). The heart rate (HR) was measured in beats per minute (bpm) with a heart rate monitor (Polar®, Kempele, Finland).

\section{Design and Procedures}

The test procedure was divided into three testing sessions on three different days (Figure 2). There was a recovery time of 48 hours between the testing sessions. Each participant was tested at a similar time of day across the three days. Thus, time-dependent changes of physiological variables in the circadian rhythm and intraday fluctuations due to ingestion and rest periods were minimized (Atkinson and Reilly, 1996; Youngstedt and O'Connor, 1999). For the first test (day 1), a special incremental treadmill ramp protocol was applied to estimate the first ventilatory threshold (VT1) (Lourenço et al., 2011; Wonisch et al., 2003). For this trial, subjects wore their own running shoes. The incline of the treadmill was constant at $1 \%$ during the test, to match the energy consumption of overground running more closely (Hanson et al., 2011; Jones and Doust, 1996). The recommended total incremental load duration between 8 and 12 minutes was applied to obtain valid values for ventilatory threshold measurements (Wonisch et al., 2003). The treadmill speed increased continuously until the 15th min time limit of the protocol was achieved, participants reached exhaustion, or abort criteria occurred, such as pain or cardiovascular problems (Steinacker et al., 2002). With the corresponding time value of VT1, the velocity of the treadmill was used to determine individual running speeds for the aerobic condition (day 2, at $70 \%$ of the individual VT1) and the anaerobic condition (day 3, at 102\% of the individual VT1).

On days 2 and 3, participants warmed up for six minutes at a running speed of $2.2 \mathrm{~m} / \mathrm{s}$ (Figure
2). Afterwards, they completed a load protocol of four running phases with duration of six minutes for each shoe condition. During the 3-min resting periods, participants changed shoes in randomized order. As recommended by Nigg et al. (2003), duration of running phases was set at six minutes to produce repeatable results. Therefore, the initial five minutes of the running phases allowed the participants to reach a steady state. Oxygen uptake (VO2), normalized to the participants' body mass $(\mathrm{ml} / \mathrm{kg} / \mathrm{min})$, and the HR were analyzed according to Divert et al. (2008) during the final $30 \mathrm{~s}$ of each shoe condition, and for the aerobic and anaerobic running phases.

\section{Statistical Analyses}

Mean and standard deviations (mean \pm SD) were calculated for all footwear-related characteristics and physiological variables. Given that variables were normally distributed according to the Shapiro-Wilk test, a one-way analysis of variance (ANOVA) followed by the Bonferroni post-hoc test was used to determine whether differences existed between mechanical midsole material characteristics. Furthermore, an ANOVA for repeated measurements followed by the Bonferroni post-hoc test was used to determine whether differences existed between physiological variables when running in different footwear conditions. Statistical significance was set at $\alpha=0.05$ for all analyses. In addition, effect size (Cohen's d) was calculated to quantify the magnitude of differences between footwear conditions and between physiological variables, if statistical significance was found. The coefficients were interpreted as trivial $(\mathrm{d}<0.2)$, small $(\mathrm{d}<0.5)$, medium $(\mathrm{d}<0.8)$, or large effect $(\mathrm{d} \geq 0.8)$ (Cohen, 1992). Relative mean differences between footwear conditions were calculated for physiological variables. Positive differences imply that higher VO2 or HR was observed for the first footwear condition analyzed. Finally, mechanical midsole characteristics were related to physiological variables with regression analyses for aerobic and anaerobic conditions.

\section{Results}

The shoe mass differences between the PM models were less than $2 \mathrm{~g}$ (Figure 1). In the rearfoot area, ANOVA revealed that stiffness and ELoss_rel were influenced by midsole characteristics, with all pair-wise comparisons 
also reaching statistical significance $(\mathrm{p}<0.001)$ (Figure 3a). Furthermore, Cohen's d showed large effects $(\mathrm{d}>0.8)$ in all footwear conditions. PM2 revealed the highest stiffness $(199.5 \pm 0.9 \mathrm{~N} / \mathrm{mm})$ and PM3 the lowest $(146.8 \pm 0.2 \mathrm{~N} / \mathrm{mm})$ (Figure 3a). Furthermore, maximum ELoss_rel was measured for PM2 (51.4 $\pm 0.1 \%)$, and minimum ELoss_rel was found for PM3 $(32.6 \pm 0.1 \%)$. The mechanical test also showed statistically significant differences between all shoes for stiffness and ELoss_rel $(p<0.001)$ for the forefoot area. Cohen's d showed large effects $(d>0.8)$ in all footwear conditions in the forefoot area as well. The midsole properties revealed that PM2 had the stiffest forefoot (stiffness: $354.8 \pm 0.4$ $\mathrm{N} / \mathrm{mm}$ ) (Figure $3 \mathrm{~b}$ ). The lowest stiffness was found for PM3 $(304.1 \pm 0.3 \mathrm{~N} / \mathrm{mm})$. In the forefoot area, similar to the rearfoot area, the maximum ELoss_rel was measured for PM2 $(39.7 \pm 0.1 \%)$, and minimum ELoss_rel was found for PM3 (29.0 $\pm 0.1 \%$ ).

For test day 1 (exhaustion test), the average maximum HR was $190.3 \pm 7.8 \mathrm{bpm}$ and average maximum VO2 was $68.8 \pm 7.9 \mathrm{ml} / \mathrm{kg} / \mathrm{min}$. Derived from the exhaustion test, the average $\mathrm{HR}$ of the participants at VT1 was $160.8 \pm 20.8 \mathrm{bpm}, \mathrm{VO} 2$ $46.5 \pm 5.6 \mathrm{ml} / \mathrm{kg} / \mathrm{min}$, and running speed $3.7 \pm 0.5$ $\mathrm{m} / \mathrm{s}$. Therefore, based on the results of day 1 , subjects ran an average of $2.6 \pm 0.2 \mathrm{~m} / \mathrm{s}(70 \%$ treadmill speed of individual VT1) in the aerobic condition on day 2 , and $3.8 \pm 0.4 \mathrm{~m} / \mathrm{s}(102 \%$ treadmill speed of individual VT1) in the anaerobic condition on day 3 . The results of $\mathrm{VO} 2$ and the HR for aerobic and anaerobic running in the different shoes are shown in Table 1, and relative mean differences between footwear conditions are shown in Table 2. For VO2 as well as for the HR, no significant differences between shoes were found for aerobic (VO2: $p=0.533$; HR: $\mathrm{p}=0.623)$ or anaerobic conditions $(\mathrm{VO} 2: \mathrm{p}=0.292$; HR: $\mathrm{p}=0.217$ ).

Results of the physiological variables showed that there was an individual trend for the participants when comparing footwear conditions. Furthermore, no significant relationships between physiological variables and footwear conditions were found (Table 3).

Table 1

Group mean \pm standard deviation of aerobic and anaerobic test sessions for oxygen uptake $\left(\mathrm{VO}_{2}\right)$ and the heart rate (HR)

\begin{tabular}{|c|c|c|c|c|c|c|c|c|c|c|c|c|}
\hline \multirow[b]{2}{*}{ shoe } & \multicolumn{3}{|c|}{$\begin{array}{l}\mathrm{VO}_{2} \text { aerobic } \\
{[\mathrm{ml} / \mathrm{kg} / \mathrm{min}]}\end{array}$} & \multicolumn{3}{|c|}{$\begin{array}{c}\mathrm{VO}_{2} \text { anaerobic } \\
{[\mathrm{ml} / \mathrm{kg} / \mathrm{min}]}\end{array}$} & \multicolumn{3}{|c|}{$\begin{array}{l}\text { HR aerobic } \\
{[\mathrm{bpm}]}\end{array}$} & \multicolumn{3}{|c|}{$\begin{array}{c}\text { HR anaerobic } \\
{[\mathrm{bpm}]}\end{array}$} \\
\hline & Mean & \pm & SD & Mean & \pm & SD & Mean & \pm & SD & Mean & \pm & SD \\
\hline PM1 & 36.4 & \pm & 3.8 & 49.8 & \pm & 5.8 & 142.2 & \pm & 16.5 & 172.7 & \pm & 12.1 \\
\hline PM2 & 36.9 & \pm & 3.3 & 50.8 & \pm & 5.7 & 142.4 & \pm & 15.6 & 173.7 & \pm & 13.5 \\
\hline PM3 & 36.9 & \pm & 3.6 & 50.4 & \pm & 5.9 & 143.0 & \pm & 16.4 & 175.8 & \pm & 14.6 \\
\hline PM4 & 36.3 & \pm & 3.3 & 49.9 & \pm & 6.4 & 144.2 & \pm & 15.4 & 172.3 & \pm & 14.7 \\
\hline
\end{tabular}

Note: no statistical significance between shoe conditions was found ( $n=15$ participants) 
Table 2

Relative mean differences between footwear conditions

\begin{tabular}{|c|c|c|c|c|}
\hline & $\begin{array}{c}\mathrm{VO}_{2} \text { aerobic } \\
{[\%]}\end{array}$ & $\mathrm{VO}_{2}$ anaerobic $[\%]$ & HR aerobic [\%] & HR anaerobic [\%] \\
\hline PM1 vs. PM2 & -1.4 & -2.1 & -0.2 & -0.6 \\
\hline PM1 vs. PM3 & -1.4 & -1.3 & -0.6 & -1.8 \\
\hline PM1 vs. PM4 & 0.2 & -0.2 & -1.4 & 0.2 \\
\hline PM2 vs. PM3 & 0.0 & 0.8 & -0.5 & -1.2 \\
\hline PM2 vs. PM4 & 1.6 & 1.9 & -1.3 & 0.8 \\
\hline PM3 vs. PM4 & 1.6 & 1.1 & -0.8 & 2.0 \\
\hline
\end{tabular}

Note: a positive difference implies that higher oxygen uptake $\left(\mathrm{VO}_{2}\right)$ or heart rate (HR) was observed for the first named footwear condition ( $n=15$ participants)

Table 3

Regression analyses $\left(R^{2}\right)$ of footwear conditions and physiological variables for aerobic and anaerobic conditions

\begin{tabular}{|c|c|c|c|c|}
\hline & & & & $\begin{array}{c}\text { HR anaerobic } \\
\mathrm{R}^{2}\end{array}$ \\
\hline & $\begin{array}{c}\mathrm{VO}_{2} \text { aerobic } \\
\mathrm{R}^{2}\end{array}$ & $\begin{array}{c}\mathrm{VO}_{2} \text { anaerobic } \\
\mathrm{R}^{2}\end{array}$ & $\begin{array}{c}\text { HR aerobic } \\
\mathrm{R}^{2}\end{array}$ & \\
\hline \multirow{3}{*}{ Stiffness rearfoot } & 0.02 & 0.23 & 0.02 & 0.12 \\
\hline & & & & \\
\hline & 0.25 & 0.03 & 0.00 & 0.79 \\
\hline \multicolumn{5}{|l|}{ ELoss_rel rearfoot } \\
\hline & 0.08 & 0.00 & 0.15 & 0.54 \\
\hline \multicolumn{5}{|l|}{ Stiffness forefoot } \\
\hline & 0.16 & 0.00 & 0.02 & 0.64 \\
\hline ELoss_rel forefoot & & & & \\
\hline
\end{tabular}

Note: $p>0.05$ for all regression analyses ( $n=15$ participants) 


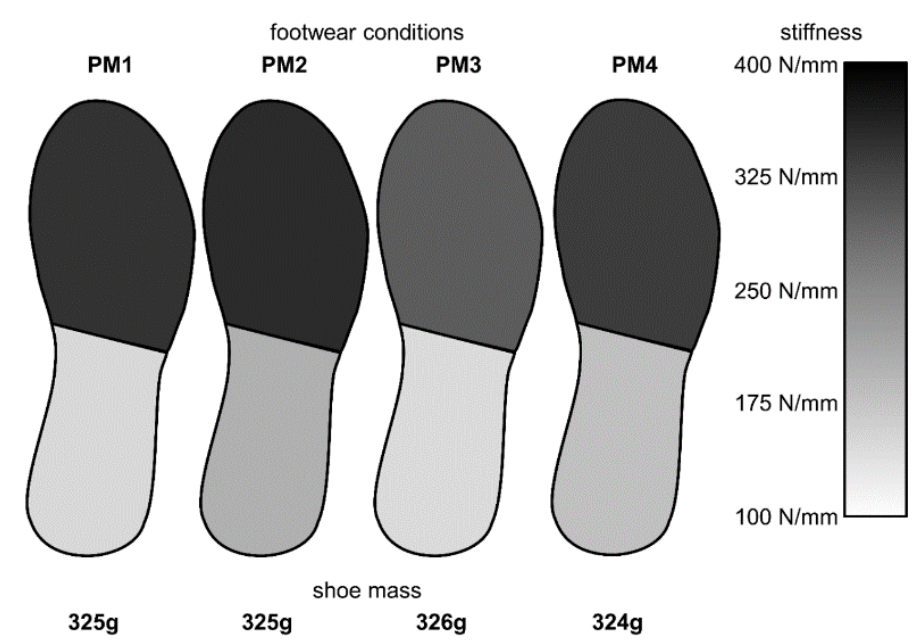

Figure 1

Stiffness for the rearfoot and forefoot areas of all footwear conditions (PM1 - PM4)

day 1 - incremental ramp protocol

* ramp protocol in accordance to Lourenço et al. (2011)

* estimation of the individual first ventilatory threshold (VT1)

* individual running shoes

recovery time of 48 hours

day 2 - aerobic condition

* warming up for 6 min at $2.2 \mathrm{~m} / \mathrm{s}$

- stage duration of $6 \mathrm{~min}$ at $70 \%$ of the individual VT1

* 4 different shoe conditions in randomized order

$*$ resting periods of 3 min between shoe conditions

recovery time of 48 hours

day 3 - anaerobic condition

* warming up for $6 \mathrm{~min}$ at $2.2 \mathrm{~m} / \mathrm{s}$

* stage duration of 6 min at $102 \%$ of the individual VT1

* 4 different shoe conditions in randomized order

$*$ resting periods of 3 min between shoe conditions

Figure 2

Test design and procedures 
a)

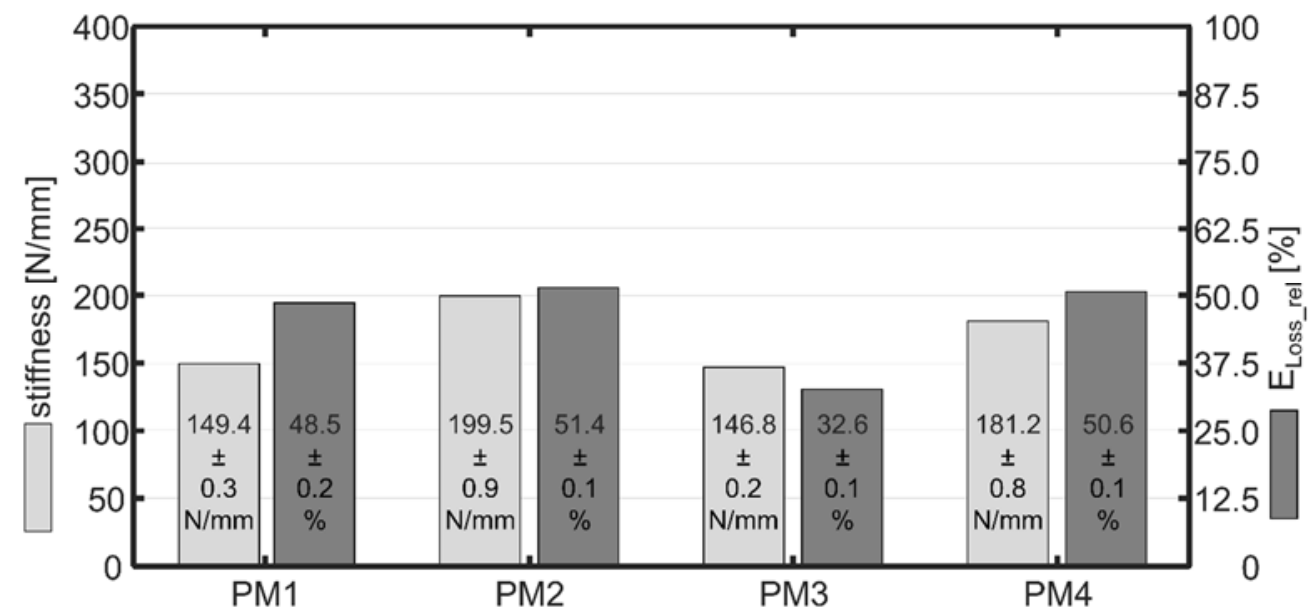

b)

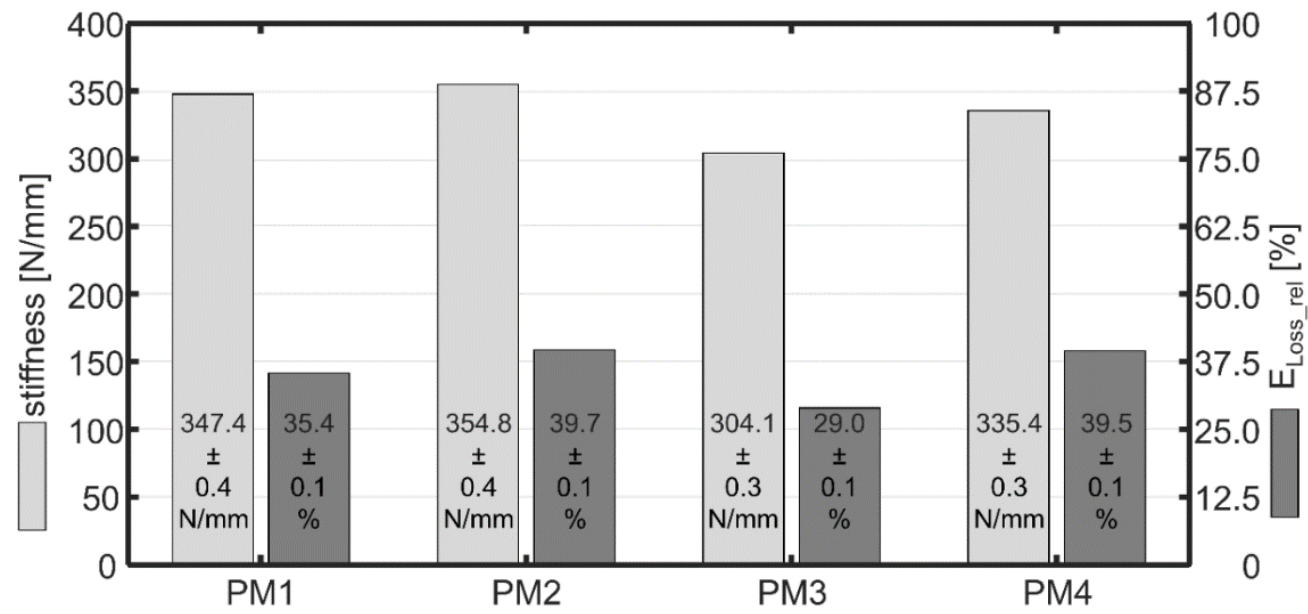

Figure 3

Mechanical properties midsole stiffness and ELos_rrel of all footwear conditions for the a) rearfoot and $b)$ forefoot area; statistical significance $(p<0.05)$ and large effects $(d>0.8)$ were found in all footwear conditions for midsole stiffness (stiffness) and relative energy loss (ELos__rel) ( $p<0.001:$ PM1 vs. PM2; PM1 vs. PM3; PM1 vs. PM4; PM2 vs. PM3;

PM2 vs. PM4; PM3 vs. PM4)

\section{Discussion}

The purpose of the present study was to determine the influence of different mechanical properties of running shoes on the physiological variables of well-trained male runners below and above athletes' individual first ventilatory thresholds (VT1). We hypothesized that running in shoes with: a) higher stiffness and b) less energy loss would lead to lower oxygen uptake and a lower heart rate during aerobic and anaerobic running.

Numerous studies have shown that different material characteristics of the shoe sole can influence performance. Nigg et al. (2003) 
investigated the influence of two shoes with different mechanical heel characteristics on oxygen uptake and EMG data while running slightly above the aerobic threshold. They found small, but non-significant mean differences for both variables when comparing shoe conditions. In addition, they stated that the harder/elastic shoe was not more advantageous to performance than the softer/visco-elastic shoe. Our results correspond with the results of Nigg et al. (2003) regarding the influence of midsole properties on physiological variables under anaerobic conditions when observing stiffness for the rearfoot and forefoot areas (Table 1). The mechanical tests of our study revealed that the stiffness of PM2 was comparable to a competition shoe, e.g. Adidas AdiStar, and stiffness of PM3 was comparable to a soft cushioned shoe, e.g. Asics Gel Nimbus 12 (Mitschke et al., 2017, 2018). However, it seems that the wide range of 52.7 $\mathrm{N} / \mathrm{mm}$ hardness differences in the rearfoot area and $50.7 \mathrm{~N} / \mathrm{mm}$ in the forefoot area (PM2 vs. PM3) was too low to influence physiological variables significantly when running slightly above the VT1, and additionally, when running below the VT1. For both running conditions, no systematic trend for the participants was found between stiffness and physiological variables, which was also supported by the results of the regression analyses (Table 3). Therefore, we had to reject hypothesis a) for aerobic and anaerobic conditions.

Regarding the energy return or energy loss of footwear, Worobets et al. (2013) studied oxygen uptake while running with two shoes with identical construction and shoe mass, but different midsole materials with varying energy loss (shoe a: $40 \%$ and shoe b: $27 \%$ ). They found significantly reduced oxygen uptake of approximately $1 \%(0.9 \%$ treadmill running and $1.1 \%$ overground running) in the shoe with less energy loss while running below the individual anaerobic threshold. Our results supplement the findings of Worobets et al. (2013). Running in the shoe with the highest ELoss_rel in the rearfoot and forefoot (PM2) was characterized on average by the highest VO2, although this was not significantly different compared to the other PM models. Similar to the findings of the influence of stiffness on physiological variables, no systematic trend for the participants was found between
ELoss_rel and physiological variables for both running conditions, which was also supported by regression analyses. Therefore, based on the findings for ELoss_rel, we have to reject hypothesis b) for aerobic and anaerobic running.

Studies investigating the influence of shoe mass on physiological variables report lower oxygen uptake between 1 and $4.5 \%$ with reduced shoe mass between 100 and $350 \mathrm{~g}$ (Catlin and Dressendorfer, 1979; Divert et al., 2008; Frederick et al., 1984; Sobhani et al., 2014). In the present study, the mass differences between the PM models were distinctly lower (maximum differences of $2 \mathrm{~g}$ per shoe). Thus, an effect of the reduced shoe mass on $\mathrm{VO} 2$ or the $\mathrm{HR}$ can be neglected.

This study has a few limitations which should be mentioned. First, the data of only fifteen out of eighteen participants could be used for analysis. Three runners were excluded from the study due to technical problems with measurement devices, which led to errors in data collection. In addition, despite the randomized order of the shoes and the three-minute resting periods between different footwear conditions, accumulated fatigue cannot be excluded for the anaerobic condition. This could be the reason for the increased variability of $\mathrm{VO} 2$ under the anaerobic condition in comparison to the aerobic runs. In addition, subjective shoe comfort was not examined. Luo et al. (2009) found a decrease in oxygen uptake of up to $1.9 \%$ for the most comfortable shoe when comparing the "most" and "least" comfortable shoe conditions. Despite identical outsoles, insoles, and upper materials of the PM shoes in our study, an effect of comfort on oxygen uptake cannot be excluded when comparing the PM shoes.

\section{Conclusion}

The results of this study show that midsole stiffness and ELoss_rel had no significant effect on runners' oxygen uptake and heart rate while running either aerobically or anaerobically. Furthermore, no significant relationships between mechanical midsole characteristics and physiological variables were found. These results may be interesting for coaches and athletes, alike. Other literature suggests that reduced shoe mass and increased comfort may be more ideal targets when trying to improve performance than 
midsole material characteristics, as found in this study. Therefore, runners should wear lightweight shoes with a high comfort rating to improve their performance.

\section{Acknowledgements}

This study has not been published and is not being considered for publication elsewhere. Partial results were presented at the $7^{\text {th }}$ World Congress of Biomechanics. The authors would like to thank Lisa Peterson for proofreading the paper as a native speaker. We would also like to thank Puma ${ }^{\circledR}$ Inc., Germany, for providing the shoes for this research project.

\section{References}

Atkinson G, Reilly T. Circadian Variation in Sports Performance. Sport Med, 1996; 21: 292-312

Brückner K, Odenwald S, Schwanitz S, Heidenfelder J, Milani TL. Polyurethane-foam midsoles in running shoes - Impact energy and damping. Procedia Eng, 2010; 2: 2789-2793

Burkett LN, Kohrt WM, Buchbinder R. Effects of shoes and foot orthotics on $\mathrm{VO}_{2}$ and selected frontal plane knee kinematics. Med Sci Sports Exerc, 1985; 17: 158-163

Catlin MJ, Dressendorfer RH. Effect Of Shoe Weight On The Energy-Cost Of Running. Med Sci Sports Exerc, 1979; $11: 80$

Cavanagh PR, Williams KR. The effect of stride length variation on oxygen uptake during distance running. Med Sci Sports Exerc, 1982; 14: 30-35

Cohen J. Quantitative Methods in Psychology. Psych Bull, 1992; 112: 155-159

Divert C, Mornieux G, Freychat P, Baly L, Mayer F, Belli A. Barefoot-Shod Running Differences: Shoe or Mass Effect? Int J Sports Med, 2008; 29: 512-518

Frederick EC, Daniels JR, Hayes JW. The effect of shoe weight on the aerobic demands of running. In: Proc World Congr Sport Med, 1984; p. 616-25

Frederick EC, Howley ET, Powers SK. Lower $\mathrm{O}_{2}$ cost while running in air cushion type shoe. Med Sci Sports Exerc, 1980; 12: 81-82

Fuller JT, Bellenger CR, Thewlis D, Tsiros MD, Buckley JD. The effect of footwear on running performance and running economy in distance runners. Sport Med, 2015; 45: 411-422

Hanson NJ, Berg K, Deka P, Meendering JR, Ryan C. Oxygen cost of running barefoot vs. running shod. Int J Sports Med, 2011; 32: 401-406

Hardin EC, Van Den Bogert AJ, Hamill J. Kinematic Adaptations during Running: Effects of Footwear, Surface, and Duration. Med Sci Sport Exerc, 2004; 36: 838-844

Jones AM, Doust JH. A 1\% treadmill grade most accurately reflects the energetic cost of outdoor running. J Sport Sci, 1996; 14: 321-327

Lourenço TF, Martins LEB, Tessutti LS, Brenzikofer R, Macedo DV. Reproducibility of an incremental treadmill $\mathrm{VO}(2)$ max test with gas exchange analysis for runners. J Strength Cond Res, 2011; 25: 19941999

Luo G, Stergiou P, Worobets J, Nigg B, Stefanyshyn D. Improved footwear comfort reduces oxygen consumption during running. Footwear Science, 2009; 1: 25-29

Mitschke C, Kiesewetter P, Milani TL. The Effect of the Accelerometer Operating Range on Biomechanical Parameters: Stride Length, Velocity, and Peak Tibial Acceleration During Running. Sensors, 2018; 18: $1-12$

Mitschke C, Öhmichen M, Milani TL. A Single Gyroscope Can Be Used to Accurately Determine Peak Eversion Velocity during Locomotion at Different Speeds and in Various Shoes. Appl Sci, 2017; 7: 659 
Nigg BM, Segesser B. Biomechanical and orthopedic concepts in sport shoe construction. Med Sci Sports Exerc, 1992; 24: 595-602

Nigg BM, Stefanyshyn D, Cole G, Stergiou P, Miller J. The effect of material characteristics of shoe soles on muscle activation and energy aspects during running. J Biomech, 2003; 36: 569-575

Perl DP, Daoud AI, Lieberman DE. Effects of footwear and strike type on running economy. Med Sci Sports Exerc, 2012; 44: 1335-1343

Schwanitz S, Odenwald S. Long-term cushioning properties of running shoes (P152). In: Eng Sport, 2008; 7. p. 95-100

Sobhani S, Bredeweg S, Dekker R, Kluitenberg B, van den Heuvel E, Hijmans J, Postema K. Rocker shoe, minimalist shoe, and standard running shoe: A comparison of running economy. J Sci Med Sport, 2014; 17: 312-316

Stefanyshyn DJ, Nigg BM. Energy aspects associated with sport shoes. Sportverletzung Sportschaden, 2000; 14: $82-89$

Steinacker JM, Liu Y, Reißnecker S. Abbruchkriterien bei der Ergometrie. Dtsch Z Sportmed, 2002; 53: 228-229

Wonisch M, Fruhwald FM, Hofmann P, Hördl R, Klein W, Kraxner W, Maier R, Pokan R, Smekal G, Watzinger N. Spiroergometrie in der Kardiologie - Grundlagen der Physiologie und Terminologie. Austrian J Cardiol, 2003; 10: 383-390

Worobets J, Tomarasa E, Wannop JW, Stefanyshyn D. Running shoe cushioning properties can influence oxygen consumption. Footwear Science, 2013; 5: 75-76

Youngstedt SD, O'Connor PJ. The Influence of Air Travel on Athletic Performance. Sport Med, 1999; 28: 197207

\section{Corresponding author:}

\section{Christian Mitschke}

Chemnitz University of Technology

Faculty of Behavioural and Social Sciences

Institute of Human Movement Science and Health

Professorship of Human Locomotion

09107 Chemnitz

Germany

Phone: +49 37153132196

Fax: +49 371531832196

E-mail: christian.mitschke@hsw.tu-chemnitz.de

ORCID: 0000-0001-7662-8062 